\title{
Capsule Commentary on Mehta et al. Race/Ethnicity and Adoption of a Population Health Management Approach to Colorectal Cancer Screening in a Community-Based Healthcare System
}

\author{
David H. Howard, PhD \\ Department of Health Policy and Management, Emory University, Atlanta, GA, USA.
}

J Gen Intern Med 31(11): 1356

DOI: $10.1007 / \mathrm{s} 11606-016-3803-2$

(c) Society of General Internal Medicine 2016

$\mathrm{W}$ hen colon cancer screening is available by patient request but is not actively offered to patients, patients who are more knowledgeable and more comfortable interacting with the health system will be more likely to receive it. ${ }^{1}$ Programs that reduce the role of patient initiative should reduce variation in screening rates due to patient characteristics such as race. Kaiser Permanente of Northern California put this theory to the test by implementing a program, including automated medical alerts and mailed FIT tests, to increase colon cancer screening rates among enrollees. ${ }^{2}$ The components of the program were consistent with evidencedbased recommendations for increasing screening by the Center for Disease Control and Prevention's Community Preventive Services Task Force. ${ }^{3}$ As a fully integrated delivery system, Kaiser has the ability to implement programs that other systems, many of which are striving to become more like Kaiser, do not. Still, many of the features of this particular program could be adopted by other health systems or large primary care practices.

Screening rates increased from $35 \%$ in 2004 to $90 \% 2013$. Screening rates in the early period will be underestimated if the investigators count a patient who received a colonoscopy or sigmoidoscopy shortly before the beginning of the study period as unscreened. Screening rates for later years in the analysis are measured using a longer look-back period. Still, even if the actual increase was slightly less than the reported increase, the result is impressive in comparison with screening rates nationally. In 2013, the colorectal cancer screening rate among individuals aged 50 to 75 nationally was only $59 \%$.

Screening rates increased among all groups, but, somewhat surprisingly, differences between non-Hispanic whites and Hispanics, blacks, and Native Americans remained or widened over time. Even though the intervention was not specifically targeted at disparities, the result is still surprising. One would think that these groups would be most likely to benefit from proactive outreach. The result highlights the challenges in ensuring that all groups in society benefit from medical technologies.

Corresponding Author: David H. Howard, PhD; Department of Health Policy and Management, Emory University, Atlanta, GA, USA (e-mail: david.howard@emory.edu).

\section{Compliance with Ethical Standards:}

Conflict of Interest: The author has no conflicts of interest with any of the material in this manuscript.

\section{REFERENCES}

1. Peterson NB, Dwyer KA, Mulvaney SA, Dietrich MS, Rothman RL. The influence of health literacy on colorectal cancer screening knowledge, beliefs and behavior. J Natl Med Assoc. 2007;99(10):1105-1112.

2. Mehta SJ, Jensen CD, Quinn VP, Schottinger JE, Zauber AG, Meester R, et al. Race/ethnicity and adoption of a population health management approach to colorectal cancer screening in a community-based healthcare system. J Gen Intern Med. doi:10.1007/s11606-016-3792-1

3. Community Preventive Services Task Force. Updated recommendations for client- and provider-oriented interventions to increase breast, cervical, and colorectal cancer screening. Am J Prev Med. 2012;43:92-96.

4. National Cancer Institute. Cancer Trends Progress Report. NIH, DHHS, Bethesda, MD, March 2015, http://progressreport.cancer.gov

Published online July 8, 2016 